\title{
Aviation Data Integration System
}

\author{
Deepak Kulkarni, Yao Wang, May Windrem ${ }^{1}$, Hemil Patel ${ }^{2}$ and Richard Keller \\ NASA Ames Research Center \\ MS 269/3 Moffett Field CA 94035 \\ kulkarni@ptolemy.arc.nasa.gov
}

\section{Introduction}

Many airlines participate in Flight Operations Quality Assurance (FOQA) programs [1] or Airline Safety Action Programs (ASAP) [2] aimed at enhancing aviation safety. When FOQA and ASAP analysts examine flight data and safety reports, they are not able to access relevant aviation data for a variety of reasons. The required data is not always available, the data is heterogeneous, or it is available via diverse protocols and APIs. This paper describes the Aviation Data Integration System (ADIS), a software system that provides integrated access to heterogeneous data, to support safety analysis done in the ASAP and FOQA programs.

The wide-ranging information of interest for safety analysis cannot be found in any single unified information source. Therefore, this information must be actively collected and assembled from a variety of different sources, and presented in a manner that supports the users' problem-solving activities. This information integration task is non-trivial and presents a variety of technical challenges. The biggest challenge in doing this kind integration is that the flight data is often "deidentified" as the identification information associated with a flight, i.e., the flight start time and call-sign is regarded as highly sensitive by the airlines. That makes it difficult to integrate other aviation data with flight data. Furthermore, even if the time information is available, matching aircraft related data without the call signs is challenging.

\section{Types of Aviation Data Available In ADIS}

The aviation data that are of interest to FOQA and ASAP analysts include:

- Flight data, flight plans and amendments

- Weather data, such as Automatic Terminal Information Service (ATIS) and Aviation Routine Weather Report (METAR) data

- Radar track point records and track deviation

- Runway Visual Range (RVR) data

- Jeppesen charts

\footnotetext{
${ }^{1}$ With Science Applications International Corporation (SAIC)

${ }^{2}$ With Science Applications International Corporation (SAIC)
} 
- Statistical summaries of recent tracks

- FAA Enhanced Traffic Management System (ETMS) data

- Controller notes

- Images, such as airport images and Satellite Infrared Reception System (SIRS) images

- Aircraft condition exceedences

- National security alerts

- Sun positions

- Safety reports from the Aviation Safety Reporting System (ASRS) and the Aviation Safety Action Partnership (ASAP)

- Risk assessments from the Bayesian Net (BN)

These data are located in disparate locations and owned by different parties. The data can be structured, unstructured, or semi-structured. Therefore, the processes used to extract the information from these sources are also different.

Of these data types, ADIS can currently collect and process weather data, radar data, RVR data, flight data and Jeppesen charts. ADIS will gradually be expanded to include the other kinds of data listed above. The following sections describe the source and processing of the data that ADIS currently provides. We will now describe these data sources in detail.

\subsubsection{Flight Data}

Flight data are recorded in the flight data recorders in the aircraft cockpits. The airlines transfer flight data from data recorders to binary files. Each record in the binary files contains the reference (elapsed) time since the flight start, as well as the latitude, longitude, and altitude of the aircraft. ADIS uses a reader that deciphers the flight data from the binary files.

The ADIP team has received flight data for Boeing 737s. The Aviation Performance Measuring System (APMS) [3, 4] flight data are for three models of Boeing 737s737-400, 737-700, and 737-900. The time recorded in the flight data recorders comes from the onboard Flight Management Computer (FMC). For Boeing 737-900 aircraft, the FMC time is updated from the GPS. For Boeing 737-400 and 737-700 model aircraft, the FMC time is set by the pilot.

To secure the data, the call signs and the flight date of every flight have been masked. The call signs have been replaced by dummy flight keys. The information about a flight and the flight data are stored in three different sources:

1. The APMS database stores the dummy flight key, origin airport, departure runway, destination airport, arriving runway, and name of the binary flight data file for each flight.

2. The APMS Full Flight Encrypted (FFE) files contain the dummy flight key and the start date and time of a flight. These files are in Extensible Markup Language 
(XML) format. The FFE files are encrypted using a public key/private key encryption mechanism.

3. The APMS Full Flight Data (FFD) files are binary files that contain the flight elapsed time in seconds and the latitude, longitude, and altitude of an aircraft.

ADIS includes software that can decrypt and retrieve all the information about a flight from all three APMS data sources.

\subsubsection{Weather Data}

ADIS captures and distributes three types of weather data:

- Automatic Terminal Information Service (ATIS) data

Air traffic controllers use ATIS to provide local weather data, hazardous weather warnings, runways in use, approach information, Notices to Airmen (NOTAMS), and advisories to pilots, such as bird activity and runway braking action or friction reports. ATIS information is used by aircraft that arrive, depart, and operate within the terminal area. ATIS data is made available to pilots via recorded voice messages. These messages are repetitively broadcast on voice outlets such as phone lines and high frequency radio channels.

Some airports are equipped with Digital ATIS (D-ATIS). At these airports, the Tower Data Link System (TDLS) receives the weather information from a site weather observation system. A controller can edit the weather and airport information. D-ATIS transmits the edited ATIS text messages via a data link to aircraft, airlines, and other users. The system uses an automated voicing dictionary to synthesize voice messages from the text messages. It broadcasts these messages over existing ATIS frequencies.

- Aviation Routine Weather Report (METAR) data

METAR is the surface aviation weather observations that cover weather elements pertinent to flying, such as wind, visibility, runway visual range, weather phenomena, sky condition, temperature, dew point, and altimeter reading. METAR is the interpretation of the local weather conditions by weather observers at a network of airport stations. METAR is used by the National Weather Service (NWS) to determine the flying category and to produce the Terminal Aerodrome Forecast (TAF).

A METAR is observed once per hour or once per half hour, depending on the airport.

- Aviation Selected Special Weather Report (SPECI) data

SPECI is a non-routine aviation weather report. It has the same format and content as METAR. SPECI is produced when changes in weather conditions meet a set of criteria, such as when a funnel cloud is observed or when the runway visual range has dropped below 2,400 feet. 
Because METAR and SPECI have the same format and are generated and distributed the same way, this paper will treat SPECI as a subset of METAR and group them under METAR. The sources of METAR and ATIS data are described in the following sections.

\subsubsection{SkySource}

SkySource is a service of ARINC. SkySource provides access to METAR, ATIS, and Terminal Aerodrome Forecasts (TAF). ARINC receives D-ATIS text messages from the air traffic control towers via the ARINC Data Network Service (ADNS). With a paid annual subscription, ADIS gets ATIS and METAR information from SkySource via the Internet. The SkySource Web site [5] provides the current ATIS and METAR data. When a new METAR record comes in from an airport, it overwrites the older record.

Since the ATIS and METAR records come in at irregular intervals, ADIS has to log onto the SkySource Web site frequently to download the records in order to capture all the data. Since only about $20 \%$ of airports are equipped with D-ATIS, the ADIP team decided to download data for those airports only. ADIS now uses SkySource for ATIS data only and obtains the METAR data from other sources. This approach allows ADIS to poll more often and, thus, retrieve a complete set of ATIS data.

\subsubsection{National Climatic Data Center (NCDC)}

NCDC archives $99 \%$ of all the National Oceanic and Atmospheric Administration (NOAA) data. NCDC receives METAR data from the National Weather Service (NWS). It parses the METAR records and archives them for domestic airports only.

ADIP paid annual subscriptions to access the Web site [6] operated by NCDC. On this Web site, a user can specify the date and the airport identifier to view or download all the METAR records for that date (based on local time) and airport. The METAR records are presented in a tabular format. Airport information, such as latitude and longitude, is also displayed.

The METAR records are available on the NCDC Web site two to five days after the meteorological observation took place. This delay and the lack of foreign airports' METAR records, make NCDC a less than ideal source of METAR data.

\subsubsection{National Weather Service (NWS)}

NWS receives METAR data from the global weather and climate community only seconds after the weather observations took place at an airport. NWS has METAR data for over 3,000 airports worldwide. NWS stores the METAR data in 24 cycle files, each of which contains one hour's data. The file that is 23 -hours old is deleted and recreated to store the current hour's METAR records. The cycle files are available through anonymous FTP [7]. Some airports send in their observations late. NWS appends the late records at the end of the cycle files.

ADIP retrieves the cycle files and archives them on a UNIX server. After the cycle files are downloaded, they are parsed and stored in a SQL Server database. The METAR records of domestic airports are parsed according to the coding standards defined in the 
Federal Meteorological Handbook [8]. The METAR records of foreign airports are parsed according to the coding standards defined in the World Meteorological Organization Manual on Codes [9].

Because of the following two factors, the ADIP team has decided to use NWS as the source of METAR data:

1. NWS provides METAR records for both domestic and foreign airports

2. NWS updates the cycle files every 5 minutes as the METAR records are received from weather stations around the world.

\subsubsection{Radar Data}

The radar data that the ADIP team received was extracted from the Performance Data Analysis and Reporting System (PDARS) [10]. PDARS was developed by Avionics Test and Analysis Corporation (ATAC) under the joint sponsorship of FAA and NASA. PDARS analyzes the radar track data to monitor, measure, analyze, and display air traffic operations performance.

The Federal Aviation Administration (FAA) gave permission to ATAC to provide ADIP one month's radar data for use in a demonstration to Alaska Airlines. ATAC provided radar data for May 2002 to ADIP. The data include both arriving and departing flights for LAX and SFO.

Because of the sensitivity of the data, the FAA instructed ATAC to mask the call signs and aircraft types for all airlines except Alaska. A PDARS-generated flight key is used to identify each flight. Three types of records are associated with each flight: flight plan, header, and track point records. ADIS does not use the flight plan records. The header records contain the origin and destination airports of a flight. The track point records contain the GMT time, latitude, longitude, altitude, and course of the aircraft.

The radar data were parsed and stored in an SQL Server database. For a single month, there were 4.3 million track point records for 30,000 flights for SFO and 12 million track point records for 54,000 flights for $\mathrm{LAX}$.

\subsubsection{Runway Visual Range Data}

The airport Runway Visual Range (RVR) system measures visibility, background luminance, and runway light intensity to determine the distance a pilot should be able to see down the runway. This distance, the runway visual range, is used to define the precision landing category of operations. The forward scatter visibility sensor, which measures the amount of light scattered by fog, snow, or freezing rain, is proven to perform over the full range of weather conditions.

The FAA's RVR site [11] has provided real-time Runway Visual Range data to the public since August 2002. The RVR system at an airport reports RVR values from 0 to 6000 feet that represent the distance a pilot is able to see down the runway. For a fully instrumented runway, RVR values are given for the touchdown, midpoint, and rollout 
portions of the runway, along with an indication of whether the values are increasing, decreasing, or stationary. In addition, the status of edge and centerline lights is given.

The FAA receives the official RVR data obtained from Air Traffic Control (ATC). The FAA Web site displays all of the current RVR data for a single airport in a table and displays the last hour's RVR data for a single runway in a graph. A user of the FAA Web site can select the frequency (every ten seconds or one minute) at which the RVR data are refreshed. The RVR value displayed is the average of the values over the last minute.

ADIP has developed software to download RVR data from the FAA Web site and to store the data in an SQL Server database.

\subsubsection{Jeppesen Charts}

Jeppesen charts consist of various images about airports that include runway images and Very High Frequency Omni-directional Range (VOR) approach charts. This information is valuable for Flight Analysts to analyze flight events and patterns. With a paid subscription, ADIP receives monthly updates of the Jeppesen charts.

Because the Jeppesen Web site [12] only provides the latest charts, ADIS archives the images for historical data retrieval.

\section{ADIS Functionality}

We developed three versions of ADIS to support airlines. The first version that has been developed to support ASAP teams provides weather and ATIS messages for a specified airport at a specific time. A second version supports FOQA teams, and provides singleclick access to weather/ATIS data relevant to FOQA analysis without requiring an user to specify date and time. The third version is a prototype that demonstrates integration of aviation data into flight data analysis programs. We will now describe these in detail.

\subsection{ADIS for ASAP users}




\section{Aviation Data Integration System (ADIS)}

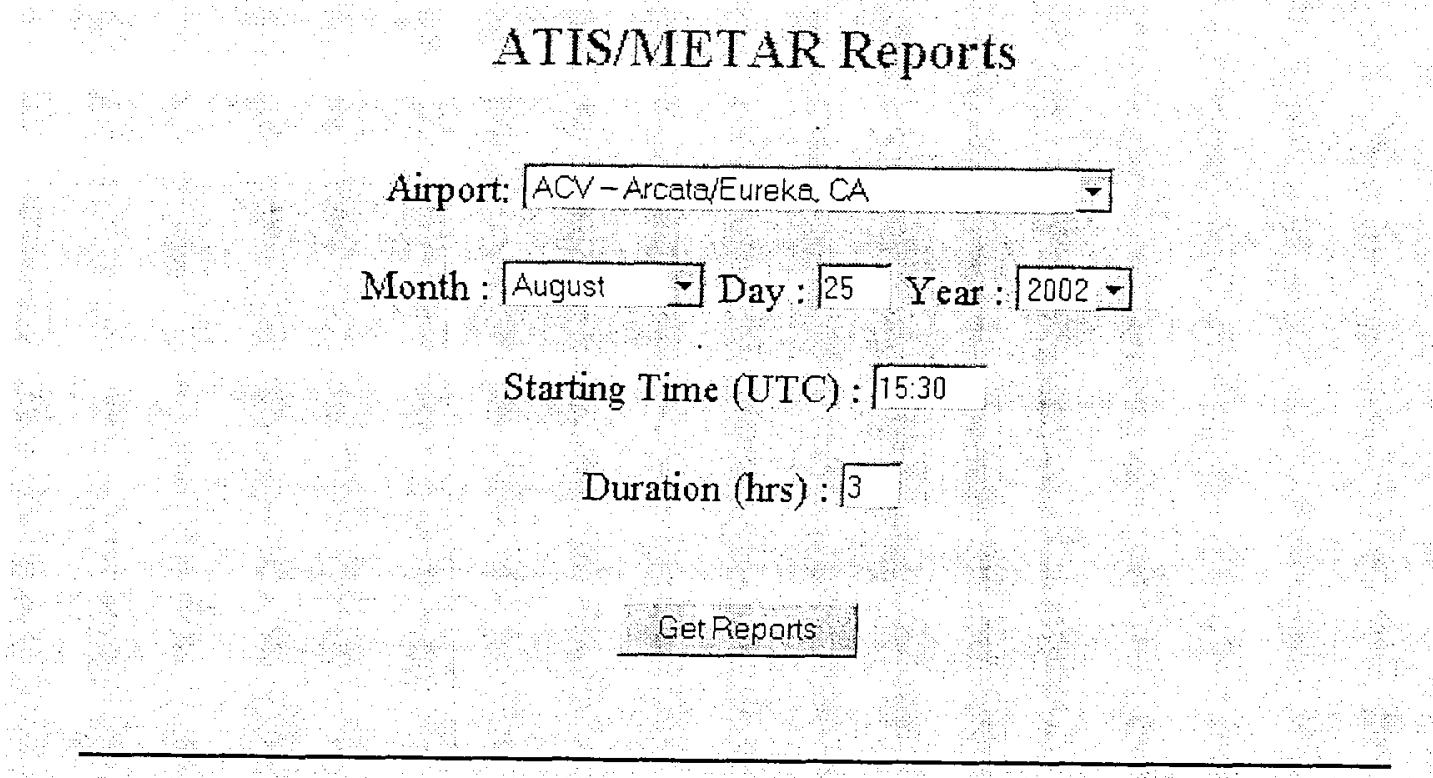

ADIS Weather Display Program is demonstration sofmare developed by NASA \& me Research Center. Please contact

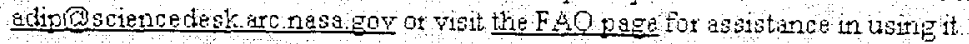

Figure 3, ADIS for ASAP users

The first version of ADIS is very simple to use. Based on the common requirements gathered from our customer airlines, we designed and implemented the ADIS Graphical User Interface (GUI). Currently, ATIS and METAR data is available on ADIS. Figure 3 shows the front page of ADIS. In this page, a user selects airport, date, time, and duration. Clicking "Get Report" button brings up the weather report page, which displays ATIS data. If ATIS data is unavailable for the selected airport at the selected time, then METAR data will be displayed. Figure 4 shows a sample weather report page. 


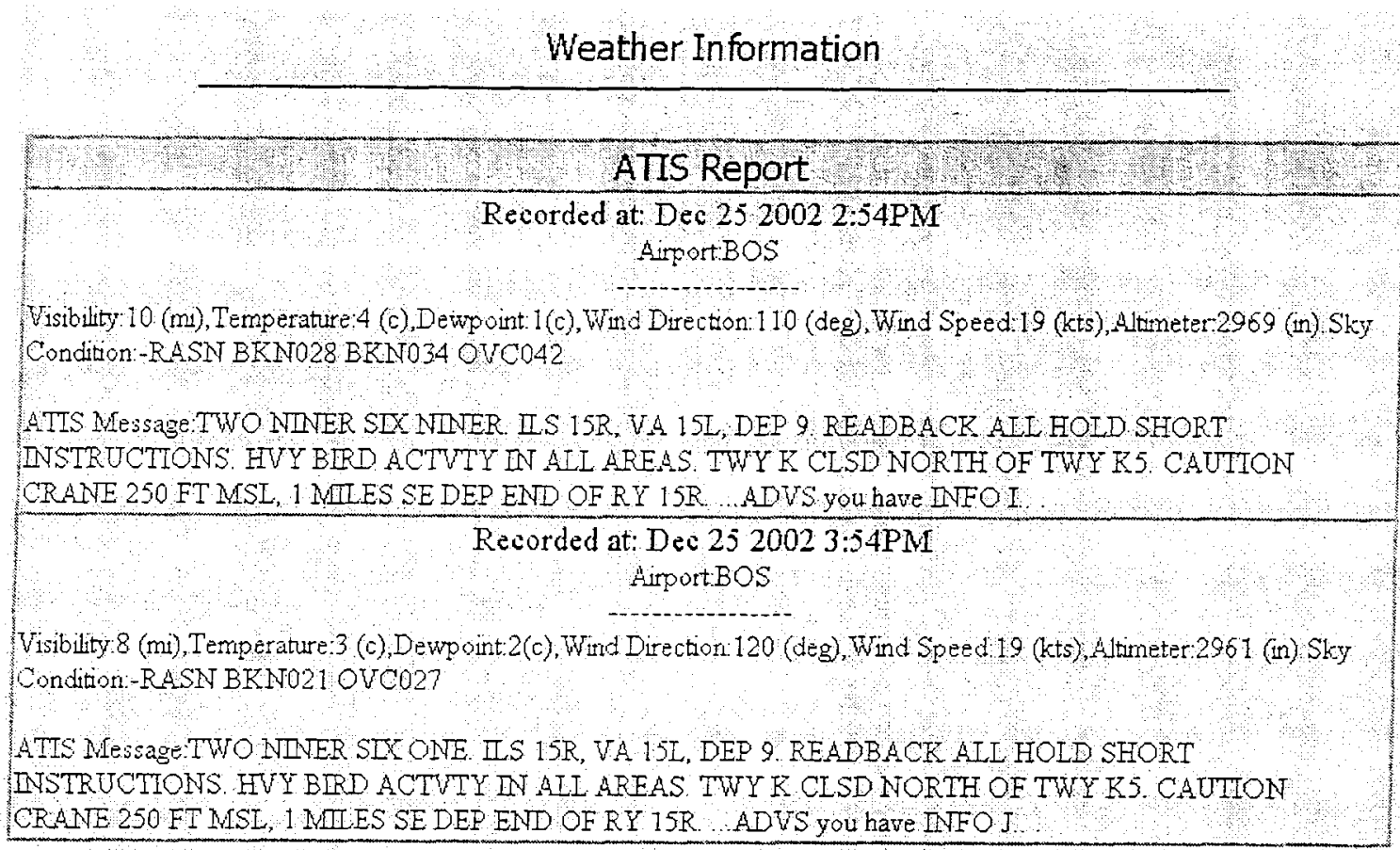

Figure 4, ADIS Weather Report

\subsection{ADIS for FOQA users}

This version of ADIS integrates aviation data with flight data while keeping the flight identification information inaccessible. This requites the implementation of security layers between data sources. In order to use the ADIS client software, the AGS program has to be running. Users can view the weather information related to the selected events/exceedances with the ADIS client (AdisWthrClnt.exe, which will have a shortcut on desktop). When ADIS is started, it displays a login dialog:

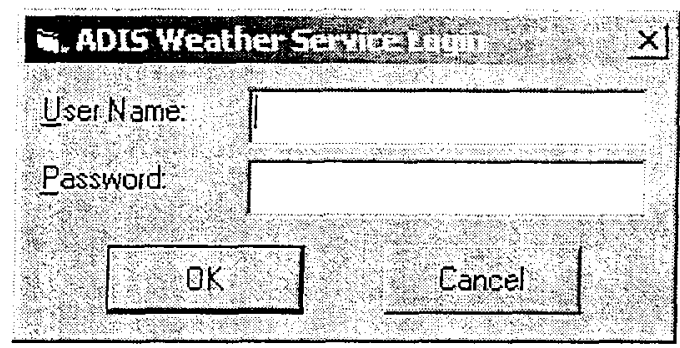

After login, the main page shows up as follows: 


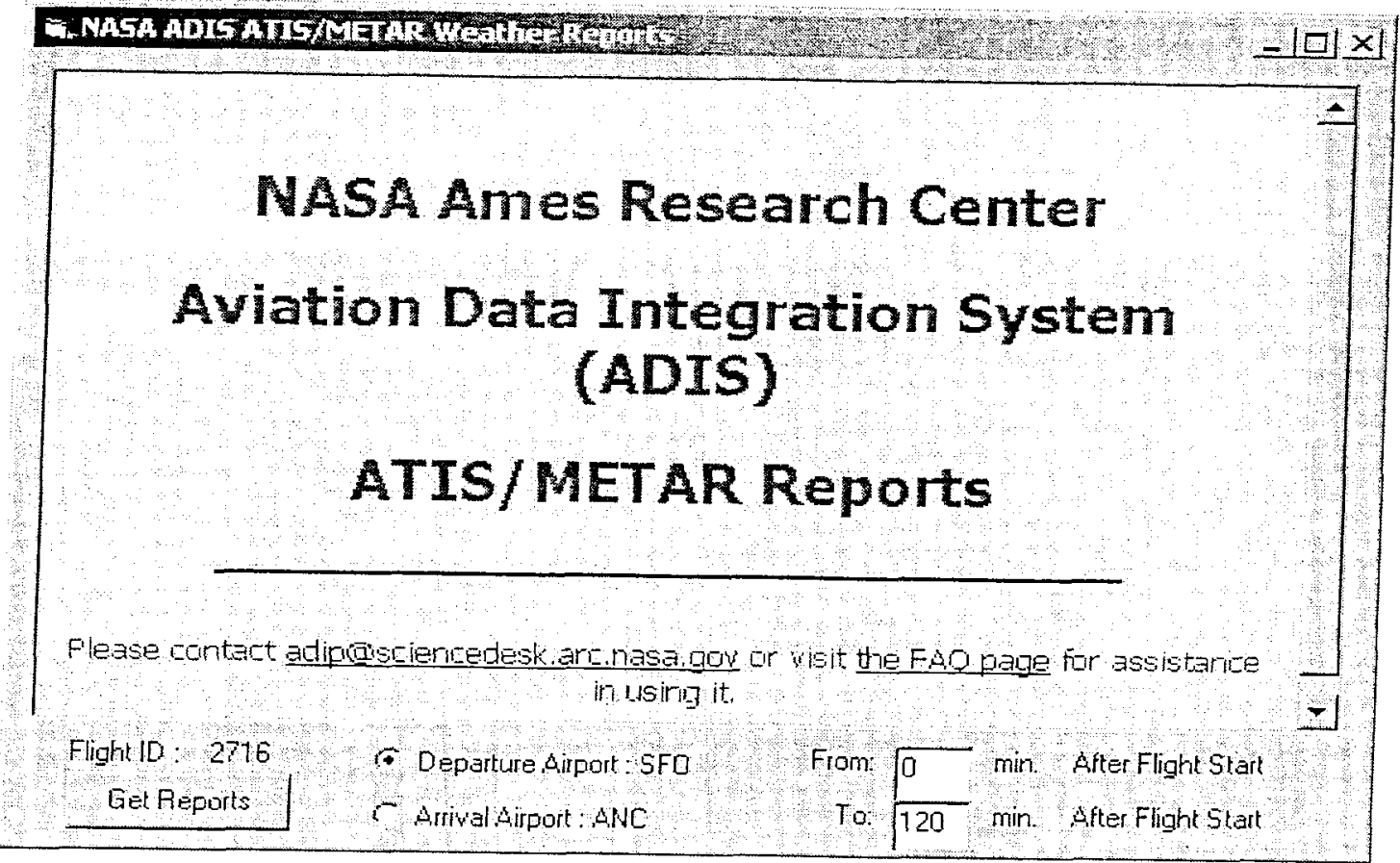

On this panel, users can select the departure or arrival airport and input the relative time interval. When the "Get Reports" button is clicked, flight-related weather and ATIS information is displayed on the screen. For the departure airport of a specified flight event, the weather (ATIS) report is displayed as follows:

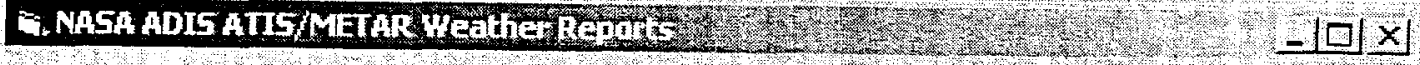

Weather Report for FlightID: 2716

Departure Airport: SFO

Time Range: From 0 To 120 Minutes After Flight Start Information Source ; ATIS

\section{Recorded at: -33 Minutes After Flight Start Departure Airport: SFO}

Visibility: 10 (mi), Temperature: 12 (c), Dewpoint:08 (c), wind Direction: 260 (deg), wind Speed: 11 ( $t 5$ ), Altimeter 0005 (in), sky Condition:

ATIS Message:THREE ZERO ZERO FIVE, ILS MW $28 R$ AFF IN IISE.

FlightID 2716

Get Reports

6 Departure Airport SFO

CAmival Airport : ANC

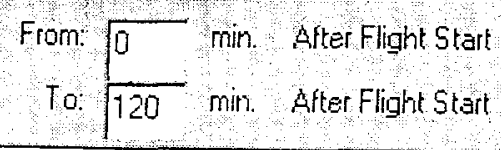


For the arrival airport, the weather (for ANC, only has METAR data) report is displayed as follows:

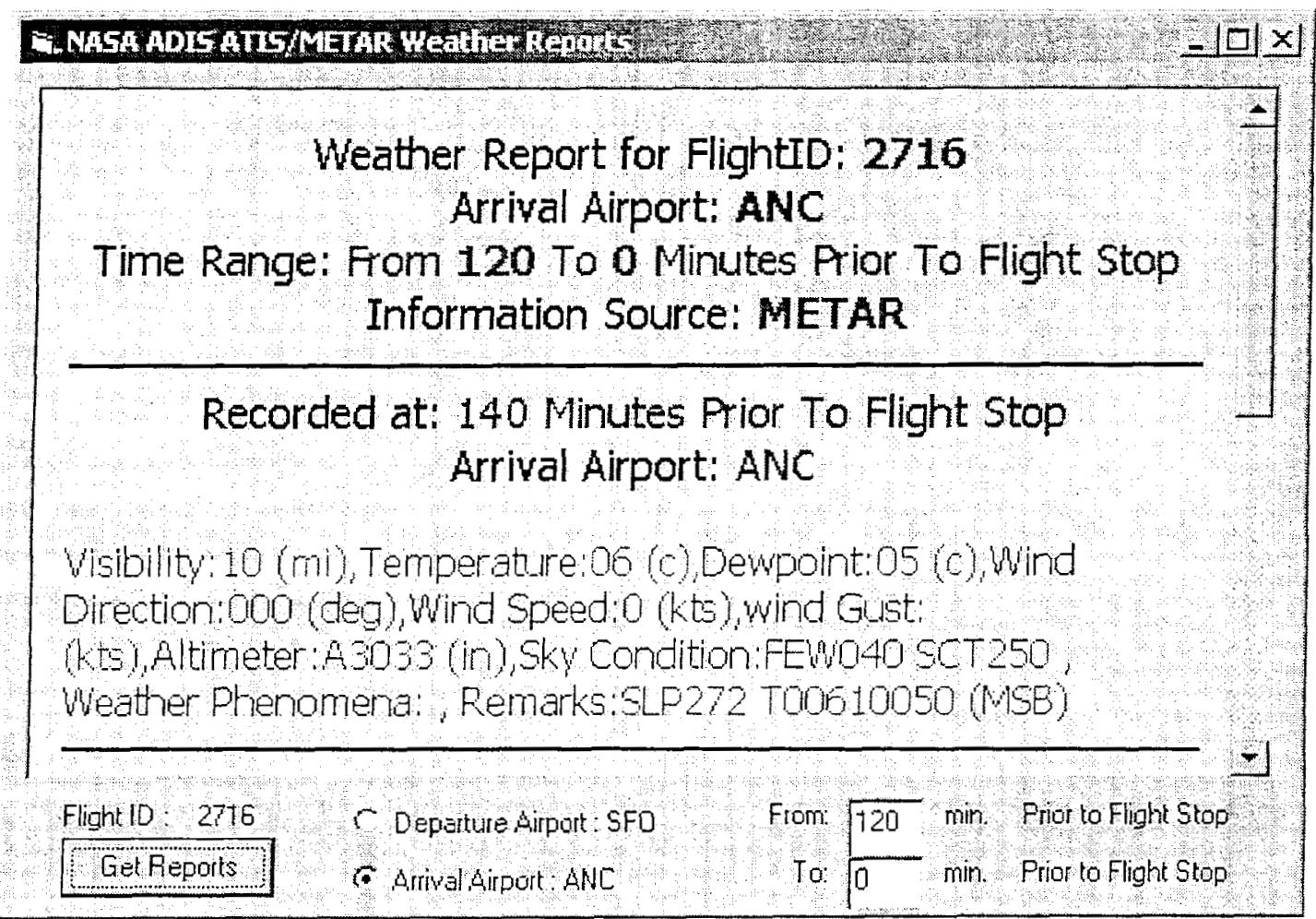

To print the report, the user can click the right mouse button and choose "Print" from the menu. To go back to the starting page, the user can choose "Refresh" on the menu.

\subsection{Prototype of ADIS Integration with flight data analysis software}

While allowing analysts to view relevant aviation data when they perform safety analysis is useful by itself, integrating it into a flight data analysis software offers additional benefits. It permits a single-click access to data relevant to a particular type of analysis. Also, it permits automated analysis of integrated data. We have developed a prototype of ADIS that is integrated with aflight data analysis software, APMS. The ADIS prototype allows flight analyst to select an APMS produced flight pattern and the analyst to search for weather patterns by displaying integrated weather, weather histograms, and integrated view of other data sources for all the flights included in the flight pattern search. It also includes new data sources like Jeppesen charts and ASRS reports.

We implemented the prototype using ASP (Active Server Pages), SQL-Server, IIS Web Server and new technologies like XML and SOAP. Also, we used FFD (Flight Data Reader) and Flipper Graph, a server-side charting tool designed by Proworks, to chart flight data on the server side. In ADIS, when client selects a flight to display, Flipper Graph creates a flight data chart as an image on the server side and sends it over to the client. 


\section{ADIS availability}

Currently, ADIS is being used by ASAP and FOQA teams in our partner airlines. FAA plans to implement the ADIS web server on its website and make it available to all U. S. airlines in the near future. NASA Technology Commercialization Office is also offering non-exclusive ADIS licenses to vendors interested in utilizing integrated data in FOQA support tools.

\section{Conclusions}

During the analysis of flight data and safety reports done in ASAP and FOQA programs, airline personnel are not able to access relevant aviation data for a variety of reasons. We have developed the Aviation Data Integration System (ADIS), a software system that provides integrated heterogeneous data to support safety analysis. Types of data available in ADIS include weather, D-ATIS, RVR, radar data, and Jeppesen charts, and flight data. We developed three versions of ADIS to support airlines. The first version has been developed to support ASAP teams. A second version supports FOQA teams, and it integrates aviation data with flight data while keeping identification information inaccessible. Finally, we developed a prototype that demonstrates the integration of aviation data into flight data analysis programs. The initial feedback from airlines is that ADIS is very useful in FOQA and ASAP analysis.

\section{References:}

[1] Flight Operational Quality Assurance Program, Code of Federal Regulations, Title 14, Volume 1, Section 13.401, U.S. Government Printing Office via GPO Access 9, 14CFR13.401, 2003

[2] The Federal Aviation Administration (FAA) Aviation Safety Action Program (ASAP). (February 28, 2003) Retrieved June 24, 2003, from http://www2.faa.gov/avr/afs/afs200/afs230/asap/

[3] Aviation Performance Measuring System (APMS). (n.d.). Retrieved June 24, 2003, from http:/human-factors.arc.nasa.gov/projects/ihs/aviationperf.html

[4] Aviation Performance Measuring System (APMS). (n.d.). Retrieved June 24, 2003, from http://www.aerospace.nasa.gov/library/chicago/apms.htm

[5] SkySource Login. (n.d.). Retrieved June 24, 2003, from http://www.skysource.net/servlet/LoginServlet

[6] NOAA National Data Centers Subscription Login. (n.d.). Retrieved June 24, 2003, from http://ols.ncdc.noaa.gov/cgi-bin/nnde/gensub.cgi 
[7] Retrieved June 24, 2003, from ftp://weather.noaa.gov/data/observations/metar/cycles

[8] Surface Weather Observations and Reports (1995), Federal Meteorological Handbook (FMH) Number 1

[9] Manual on Codes International Codes (1995), World Meteorological Organization, WMO-No. 306

[10] Performance Data Analysis and Recording System (PDARS) (n.d.). Retrieved June 24, 2003, from http://avsp.larc.nasa.gov/images asmm PDARS.html

[11] Runway Visual Range (RVR). (n.d.). Retrieved June 24, 2003, from http://rvr.fly.faa.gov

[12] Jeppesen Home Page. (n.d.). Retrieved June 24, 2003, from http://www.jeppesen.com/wlcs/index.jsp 